\title{
Effect of permanent magnets on plasma confinement and ion beam properties in a double layer helicon plasma source
}

\author{
Erik Varberg ${ }^{1, \dagger}$ and Åshild Fredriksen ${ }^{1}$ \\ ${ }^{1}$ Department of Physics and Technology, UiT The Arctic University of Norway, 9019, Troms $\varnothing$, Norway
}

(Received 29 January 2019; revised 27 May 2019; accepted 28 May 2019)

The work described in this article was carried out to investigate how permanent magnets (PM) affect the plasma confinement and ion beam properties in an inductively coupled plasma which expands from a helicon source. The cylindrical plasma device Njord has a $13 \mathrm{~cm}$ long and $20 \mathrm{~cm}$ wide stainless steel port connecting the source chamber and the diffusion chamber. The source chamber has an axial magnetic field produced by two coils, with magnetic field lines expanding into the diffusion chamber. Simulations have shown that the field lines leaving the edge of the source hit the port wall, causing a loss of electrons in this section. In the experiments performed in this work, PMs were added around the port walls near the exit of a plasma source and the effect was investigated experimentally by means of a retarding field energy analyser probe. The plasma potential, ion density and ion beam parameters were estimated, and the results with and without the PMs were compared. The results showed that the plasma density in the centre can in some cases be doubled, and the density at the edges of the plasma increased significantly with PMs in place. Although the plasma potential was slightly affected, and the beam velocity dropped by $\sim 10 \%$, the ion beam flux increased by a factor of 1.5 .

Key words: plasma applications, plasma devices, plasma diagnostics

\section{Introduction}

In inductively coupled helicon discharges (Yun, Kim \& Chang 1998), an ion beam can, in certain parameter regimes, form spontaneously at the intersection between the plasma source and the expansion chamber (Charles 2004; Charles \& Boswell 2004; Corr et al. 2007; Virko et al. 2007; Byhring et al. 2008). For this reason, these sources have for some time been studied for the purpose of developing ion thrusters for spacecraft (Charles 2009). The geometry in which the plasma expands from the source into the diffusion chamber plays an important role in shaping the sharp potential drop, a so-called current-free double layer (CFDL), which is commonly observed to form an ion beam in helicon device (Charles 2004; Virko et al. 2007). A main part of this geometry is the magnetic field. Typically, helicon sources are operated with an axial magnetic field produced with DC current coils around a

$\dagger$ Email address for correspondence: erik.varberg@outlook.com 
cylindrical source chamber, with field lines expanding from the source into the diffusion chamber (Charles 2007). The expanding field lines are effectively guiding the electrons radially outwards, while ions with a much larger Larmor radius have been found to detach at approximately $51 \mathrm{~cm}$ (Gulbrandsen et al. 2015). This radial charge separation sets up electric fields which tend to diverge the ion beam and hence deteriorate the thrust.

For thruster applications, a substantial amount of work has been carried out in order to understand the physical mechanisms of plasma expansion from a so-called magnetic nozzle towards a lower magnetic field and detachment (Schoenberg et al. 1993; Cohen et al. 2006; Olsen et al. 2015; Takahashi \& Ando 2017). Magnetic nozzles are sometimes referred to as the plasma propulsion analogue to Laval nozzles for combustion-type propulsion (Ebersohn et al. 2014). Instead of the walls in the Laval nozzle guiding the expelled matter, the magnetic nozzle guides the charged plasma particles along the magnetic field lines.

As coils along with their power supplies are heavy and bulky and thus not desirable for lightweight spacecraft payloads, permanent magnets (PMs) have been investigated as an option to provide the necessary field geometry (Chen 2008; Takahashi et al. 2008, 2009). The use of magnetic dipoles in the form of PMs along the wall of a plasma chamber has in many cases been applied as a method of confinement. Earlier experiments involving PMs in a multipole configuration have investigated their feasibility for plasma confinement and effects on low-pressure plasmas (Ohkawa \& Kerst 1961). Leung et al. (1978) discovered that the plasma confinement depends strongly on the geometry of a multipole, affecting electrons the most. Electrons with energies higher than the potential drop in the sheath are more efficiently confined, and can be used as an ionisation source for a discharge if the mean free path of these electrons is large compared to the discharge size. Also, the plasma confinement has been improved, both in radial plasma uniformity and in low-temperature bulk plasma in a discharge. Takahashi et al. (2008), Takahashi, Shida \& Fujiwara (2010), Takahashi, Itoh \& Fujiwara (2011) applied PMs to produce an axial magnetic field configuration in an expanding plasma and detected accelerated ions at supersonic speeds with increasing magnetic field strength. Chen (2012) used PMs from an axial magnetic field source for a small helicon source inside a large chamber in order to find a better antenna coupling, and it was found to produce a higher density than expected (Chen \& Torreblanca 2009).

In this work we study how PMs placed around the rim of the port between the source chamber to the diffusion chamber affect the downstream plasma and ion beam. Calculations of the expanding magnetic field show that the field lines leaving the edge of the source are crossing the port wall (Fredriksen, Mishra \& Byhring 2010). These field lines, in particular those emerging from the outer radial edge of the source, will guide the electron population at these lines towards the port wall of the chamber, causing a loss of plasma before it reaches the diffusion chamber. As most of the radio wave power is deposited in electrons close to the wall, this means that a large portion of the hottest electrons, playing an important role in the ionisation, are lost on the port wall.

By adding PMs around the outer circumference of the port, electrons are partially blocked from hitting the port walls, and are instead deflected back into the plasma stream within the diverging field. At the same time the interior of the magnetic nozzle is not significantly altered. We have investigated the ion density and ion beam properties with and without PMs around the port wall, and from these parameters the effect on the ion beam flux was calculated. 
In $\S 2$, a description of the Njord device and the applied magnetic cusp field is given. Section 3 provides a description of the data acquisition and analysis. In $\S 4$, the results and a brief discussion are given, and the conclusions are presented in $\S 5$.

\section{Plasma device and magnetic multipole}

\subsection{The Njord device}

The Njord device is used to study plasma flows and beam formation (Byhring et al. 2008). The source chamber, made of a $30 \mathrm{~cm}$ long Pyrex glass cylinder with an outer radius of $r=6.9 \mathrm{~cm}$ and encased in an equally long aluminium casing is coupled to a diffusion chamber through an extended port with a radius of $10 \mathrm{~cm}$ and a dome, shown in figure $1(a)$.

The dome starts with a radius of $10 \mathrm{~cm}$ and ends at a $30 \mathrm{~cm}$ radius, coupled to the diffusion chamber. Four 40CF ConFlat ports are placed on the side of the dome where a probe through feed is inserted into the chamber in order to acquire data. The diffusion chamber has an outer radius of $30 \mathrm{~cm}$ and is $1.2 \mathrm{~m}$ long.

Argon gas is applied at the end of the source tube, which is closed by a grounded aluminium plate. The chamber can be pumped to a base pressure of $\sim 10^{-3} \mu \mathrm{Bar}$ by a two-pump system consisting of a Boc Edwards E2M28 rotary pump and a Leybold 361C turbomolecular pump. The pressure $P$ was monitored by a gauge from a MKS Baratron Capacitance Manometer as it is intended for the intermediate pressure range used for plasma operation. The pressures measured by this gauge was monitored at $P=0.65 \mu$ Bar at flow $Q=2.0 \mathrm{SCCM}, P=[0.82-0.85] \mu \mathrm{Bar}$ at $Q=3.0$ SCCM and $P=[1.0-1.05] \mu$ Bar at $Q=4.0 \mathrm{SCCM}$, where SCCM is the standard cubic centimeter per minute unit $\left(\mathrm{cm}^{3} \mathrm{~m}^{-1}\right)$, assuming $1 \mathrm{~atm}$ gas pressure and at $T=273 \mathrm{~K}$. At these pressures, an ion beam could be registered by a retarding field energy analyser (RFEA) probe (details of the probe will be discussed in §3). The argon gas is ionised by applying high amplitude $13.56 \mathrm{MHz}$ radio frequency (RF) waves powered by a TRUMPF Hüttinger RF generator through a helicon saddle antenna wrapped around the Pyrex glass tube. A software program made for the RF generator was used to set the forward RF-power $P_{I n}$ in the range [100-800] W in steps of $100 \mathrm{~W}$, and both $P_{I n}$, the reflected power $R_{R e f}$ and the voltage standing wave ratio (VSWR) were monitored. During operation and changes of the RF-power, pressure and coil current values, the antenna was tuned by a $\Pi$-tuning network to keep the reflected power $P_{\text {Ref }}$ as close as possible to a minimum (below $5 \%$ ). The axial magnetic field is applied by a set of two coils with a radius of $12 \mathrm{~cm}$, length of $9.5 \mathrm{~cm}$, depth layer of $2 \mathrm{~cm}$ and their centres placed $21 \mathrm{~cm}$ apart. The reference point $(z=0 \mathrm{~cm})$ was chosen at the back plate of the source (see figure $1 a)$. The two coils were placed around the source chamber and antenna. The magnetic field of the coils was calculated with a program developed in house, which has been calibrated with Hall probe measurements. The field is calculated, based on the input parameters describing the geometry, number of windings and current in the windings. At a coil current of $I_{\text {Coils }}=6 \mathrm{~A}$, the magnetic field strength reaches a maximum of 200 Gauss on the axis $(r=0 \mathrm{~cm})$ and a strength of $\sim 156$ Gauss at the centre of the antenna (axial distance at $19 \mathrm{~cm}$ ). The magnetic field strength was set in the range of $I_{\text {Coils }}=[0-6] \mathrm{A}$, where an increase of $1 \mathrm{~A}$ on both coils increases the total axial field strength of $\sim$ [33-35] Gauss. Calculations of the induced magnetic field strength at different radii is shown in figure $1(b)$. The magnetic field is calculated on a $30 \times 30$ grid in cylindrical coordinates with a radial and axial range set at $[0-20] \mathrm{cm}$ and $[-5-60] \mathrm{cm}$, respectively, which results in a radial resolution of $1.2 \mathrm{~cm}$ and 

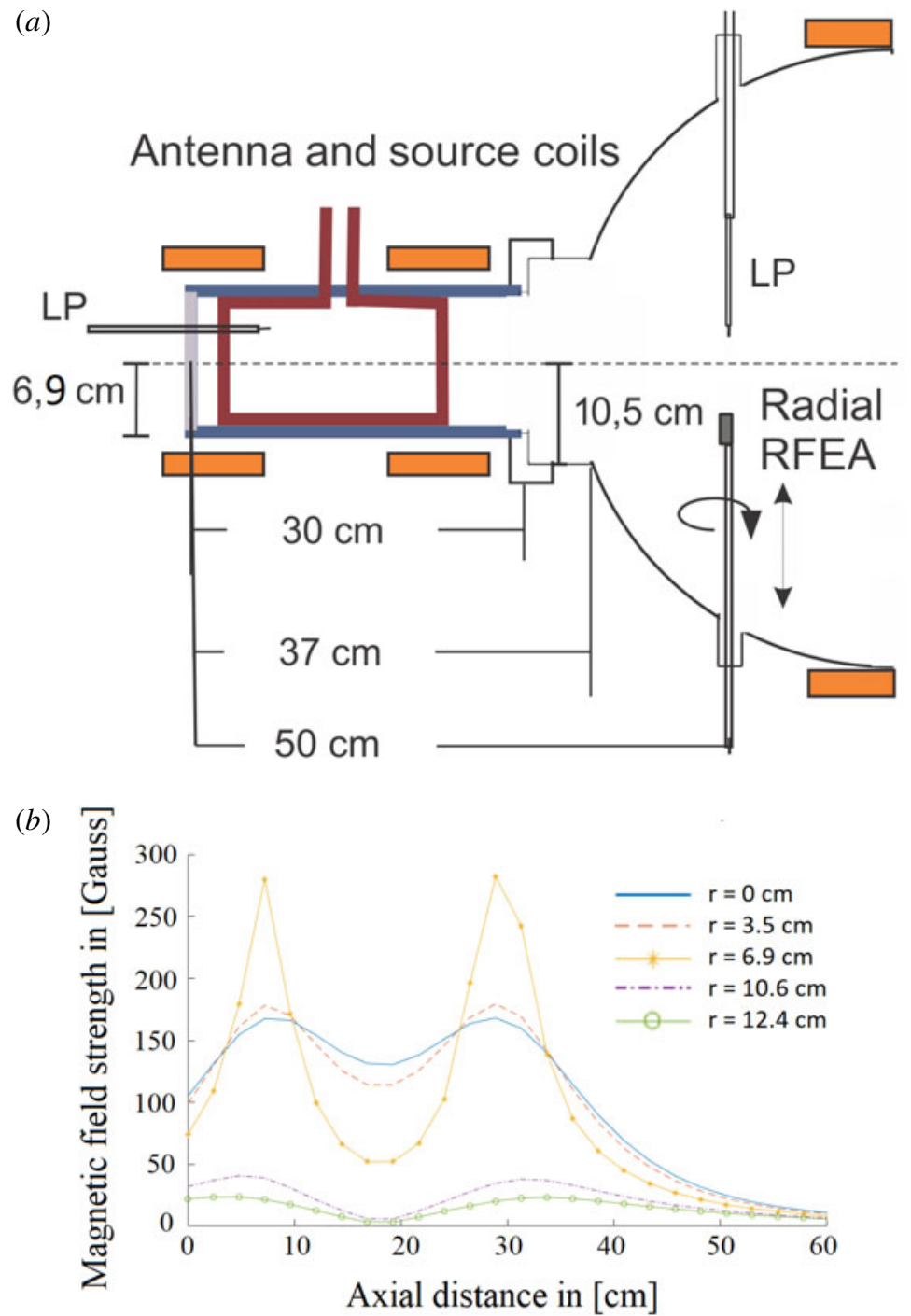

Figure 1. (a) An overview of the Njord device and its dimensions and $(b)$ a simulation of the axial magnetic field strength induced by the coils at different radial values at coil current $I_{\text {Coils }}=5 \mathrm{~A}$, which includes the centre, the outer radius of the Pyrex tube $(r=$ $6.9 \mathrm{~cm})$, the inlet chamber $(r=10.5 \mathrm{~cm})$ and at the position of the coils $(r=12.4 \mathrm{~cm})$.

axial resolution of $2.2 \mathrm{~cm}$. Although the resolution is not very high, it illustrates the structure and the field strength of the axial magnetic field.

When the plasma expands into the diffusion chamber, the inductively coupled RF powered discharge can spontaneously set up a region within the plasma with a stationary localised potential drop, known as a current free double layer (CFDL) (Byhring et al. 2008). A double layer (DL) can be described as an intersection between two local, equally oppositely charged, space charge regions within a plasma, positioned close to each other (Chen 2014). The potential drop between the regions gives rise to an electric field $E$ that depends on the distance between the regions. 
$E$ is much stronger on the inside of the DL than outside, meaning that integration of all positive and negative charges nearly cancel each other. DLs can be found in a plasma where a strong flux of charge carriers is present, which is necessary for DLs to exist. There are different types of DLs with different characteristics. They can be stationary or moving, collisional or collisionless, with a current or current free. The DL of interest here is the one formed in Njord, where the dense plasma produced in the source is expanding into the diffusion chamber along with the expanding magnetic field lines. The potential drop resulting from the decrease in plasma density can accelerate the ions and form a beam of ions on the low-potential side of the DL. As this DL does not rely on external current sources, it is commonly named a CFDL.

\subsection{Magnetic multipole confinement}

A magnetic multipole, or a multidipole, can be described as a system which consists of a series of dipoles placed side by side and alternating in the direction of the magnetic field by changing the direction of their magnetic poles. The row of magnetic fields forms a cusp configuration where the magnetic field strength is at its maximum right on the dipole and decays exponentially away from it. This creates a cross-section which is mostly free of any magnetic fields except at the boundaries of the cross-section. This field can reduce or remove the loss of plasma, increasing the plasma confinement (Lieberman \& Lichtenberg 2005).

A multipole system can be described by using a Cartesian system for a row of magnets with a width $\Delta$ in a straight line with distance $d$ between them, where $d>\Delta$ (Lieberman \& Lichtenberg 2005). If the $x$-direction represents the line where the magnets lie and the $y$-direction is the direction normal to the magnets, then the magnetic field can be described in the form of

$$
\boldsymbol{B}(x, y)=A_{0} \boldsymbol{f}(x) \mathrm{e}^{-k y},
$$

where $A_{0}$ is a constant, $f(x)$ describes the variation of the magnetic field in the $x$ direction and the last term describes an exponential decay in the $y$-direction with the decay constant $k$. The two terms are linearly independent of each other, and thus Maxwell's laws for a constant magnetic field need only be applied to $f$ as long as $y \geqslant 0$. This means that $\boldsymbol{B}$ satisfies Laplace's equation for $y \geqslant 0$, which gives

$$
\boldsymbol{B}(x, y)=\frac{2 \Delta B_{0}}{d}[\sin (\alpha x) \hat{\boldsymbol{x}}-\cos (\alpha x) \hat{\boldsymbol{y}}] \mathrm{e}^{-k y},
$$

where $B_{0}$ is the magnetic field strength at the magnet. The coefficients $\alpha$ and $k$ are usually determined by the boundary value conditions of the model.

The multidipole array used on Njord consists of a $72 \mathrm{~cm}$ long stainless steel assembly band with 18 evenly spaced neodymium magnets (Grade N42, Ring, $O D=7 / 8$ inch, $I D=1 / 4$ inch, thickness $=1 / 8$ inch), with \#8 Countersink holes, through which each magnet is fastened by stainless steel bolts to the band. It is placed around the source port of the dome at the axial position $z=37 \mathrm{~cm}, 7 \mathrm{~cm}$ away from the exit of the source chamber (the end of the Pyrex glass tube), and with a radius $r=$ $11.5 \mathrm{~cm}$. In order to retain an even distance of the cusps to the port wall, six ceramic extension pads placed every $12 \mathrm{~cm}$ along the inside of the band are supporting the assembly. Figure $2(a)$ shows the band installed on the port wall, and figure $2(b)$ shows a simulation of the magnetic field with the added field of the magnet band, 
(a)

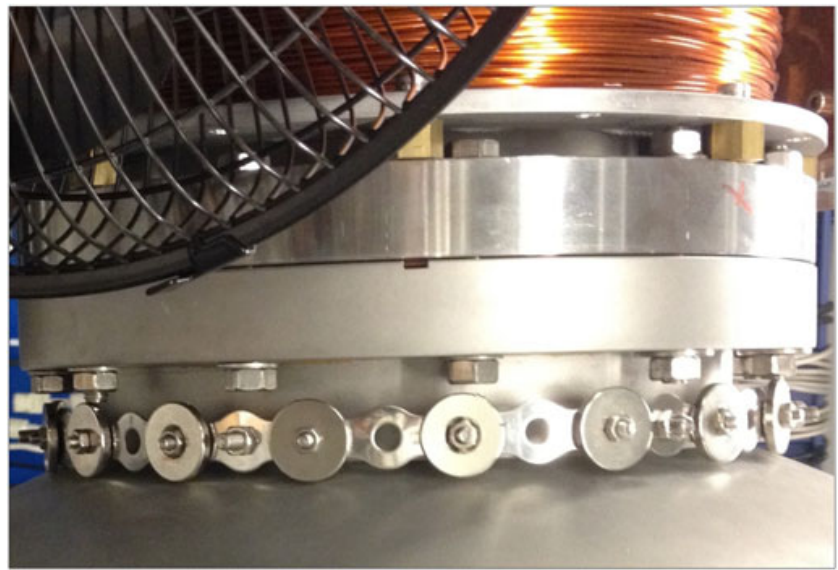

(b)

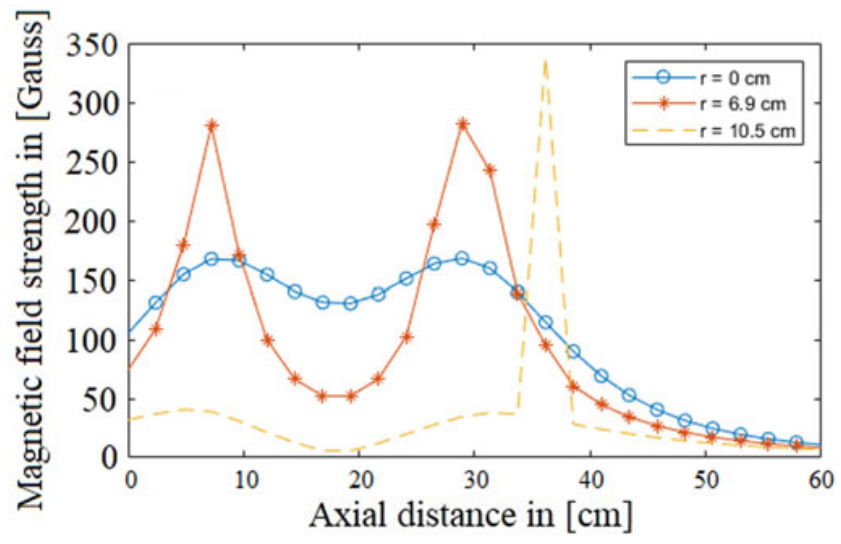

(c)

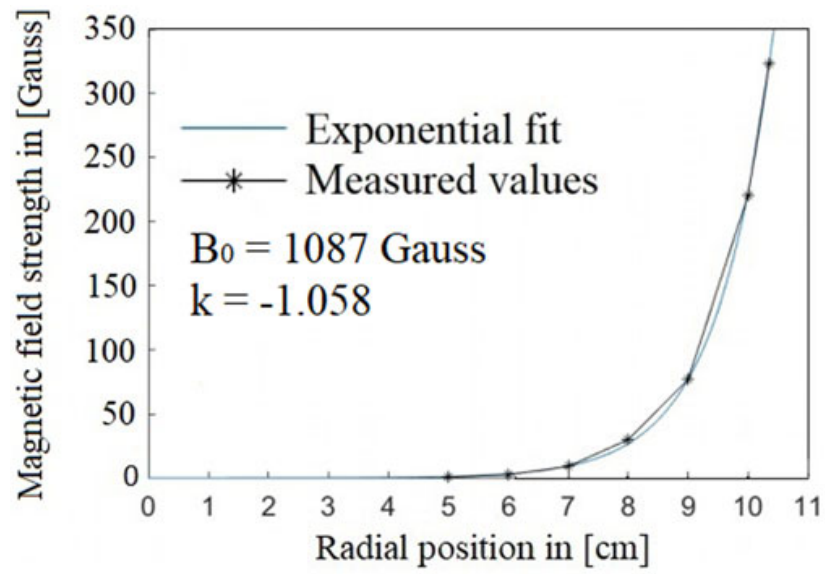

FIgURE 2. (a) The magnetic cusp band installed on Njord, $(b)$ the magnetic field configuration in axial direction at $I_{\text {Coils }}=5 \mathrm{~A}$ on each coil with the PMs on at (i) the centre of Njord $(r=0 \mathrm{~cm})$, (ii) the outer radius of the Pyrex tube $(r=6.9 \mathrm{~cm})$ and (iii) at the inner wall of the inlet port $(r=10.5 \mathrm{~cm})$ and $(c)$ graph of the fitted exponential decaying magnetic field directly on the PMs at $z=37 \mathrm{~cm}$, where $r=0 \mathrm{~cm}$ is on the cylindrical axis. Reference point of $B_{0}$ is at $r=11.5 \mathrm{~cm}$. 
(a)

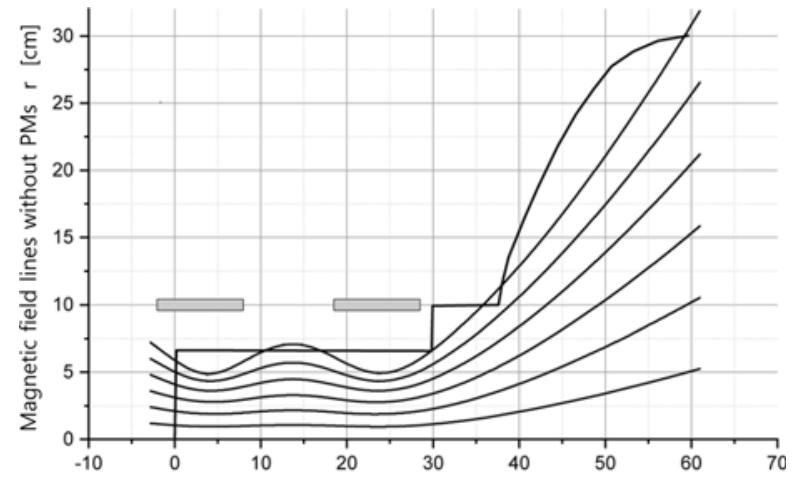

(b)

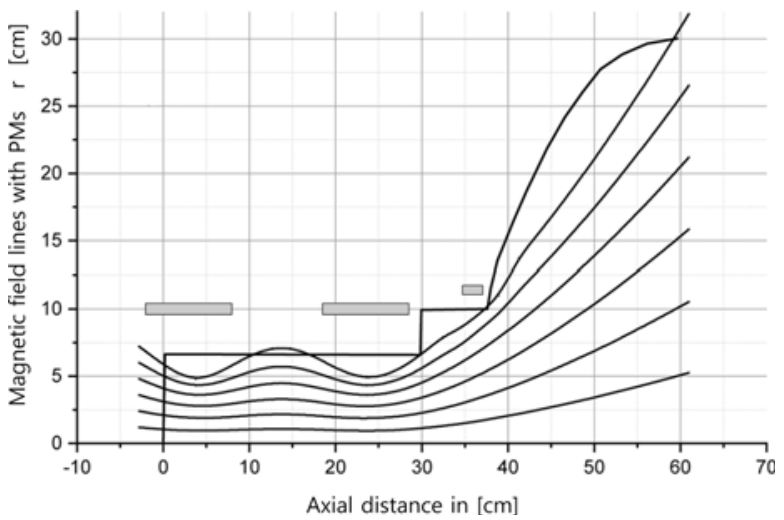

FIgURE 3. (a) Field lines calculated from the total field strength without the PMs and (b) with the PMs.

at $r=10.5 \mathrm{~cm}$ inside the port wall, done with the same simulation method as shown in figure 1(b). Figure 2(c) shows a simulation of the decaying magnetic field strength away from the magnet.

The coefficients $B_{0}$ and $k$ were found experimentally by using a gauss meter to measure the magnetic field strength both directly on the magnets and half-way between them. Six measurements at six radial positions were performed in the azimuthal direction and an average value was estimated and used in order to calculate the coefficients. They were evaluated using a first degree exponential fit in MATLAB. The results showed that directly on the PMs and perpendicular between them, the average value of the coefficients were estimated to $B_{0}=1087$ Gauss and $k=-1.058$, $B_{0}=585$ Gauss and $k=-0.8968$, respectively.

Using the total field strength and assuming the field is nearly homogeneous in the radial direction, the axial magnetic field lines without and with the PMs were calculated, and are shown in figures $3(a)$ and $3(b)$, respectively.

The area $A_{0}=\pi r_{i}^{2}$ of the cylindrical cross-section is calculated at the radius $r=r_{i}$, with $r_{i}$ obtained from the grid points in the range [1.2-7.2] $\mathrm{cm}$ of the B-field calculations and at the axial point $z_{0}=-5 \mathrm{~cm}$. With the B-field taken as $B_{0 i}$ in these grid points, flux conservation $B_{0 i}\left(r_{i}, z_{0}\right) A_{i 0}=B(r i, z) A_{i}(z)$ is used to obtain $A_{i}(z)$. Knowing $A_{i}(z)$ in a cylindrical geometry, we obtain $r_{i}(z)=\sqrt{A_{i}(z) / \pi}$, and $r_{i}(z)$ is then plotted as a representation of the field line starting at $r_{i}$. This method has also been applied in previous works (Gulbrandsen \& Fredriksen 2017). The field lines 


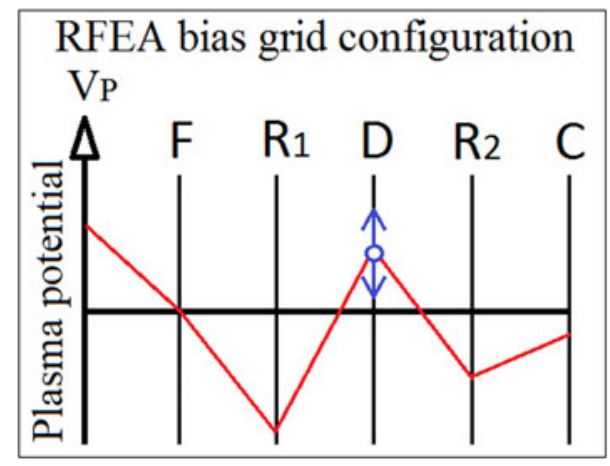

FIGURE 4. Overview of the biased grid configuration used in Njord.

without permanent magnets shown in figure 3(a) were calculated by this method. An outline of the chamber walls and placement of the solenoids are also shown. In this configuration, the magnetic field lines from near the radius of the source will hit (or graze) the port wall. We do not currently have available software to calculate magnetic fields directly from a combination of solenoids and permanent magnets. On the other hand, the field from the PMs was well known from Hall probe measurements, and this total magnetic field was added into the result matrix from the calculations of the solenoids. This procedure enables us to plot approximated field lines, but cannot render the details of the dipole fields near the surface of the port. In figure $3(b)$ the PM field is shown to bend the outermost field lines slightly, enough to reduce the direct loss of plasma particles to the port wall. A word of caution here is that as one approaches the radial position of the PMs, the magnetic field is no longer homogeneous in the radial direction, and thus the effect from, the PMs is underestimated.

\section{RFEA diagnostic}

The plasma parameters in Njord were obtained by an electrostatic retarding field energy analyser (RFEA) probe (Stenzel et al. 1982; Hutchinson 1987). The RFEA probe consists of a ceramic housing, $35 \mathrm{~mm}$ long and $23 \mathrm{~mm}$ wide, with a floating aperture grid in the front with a diameter of $6 \mathrm{~mm}$. The four grids are made from stainless steel mesh with a transmission factor of $44 \%$ and the meshes are spot welded to $0.3 \mathrm{~mm}$ thick brass spacers with $0.3 \mathrm{~mm}$ ceramic spacers between them, resulting in a distance of $0.6 \mathrm{~mm}$ between the grids. Figure 4 shows the grid configuration which was used in the experiments.

The two repeller grids $R_{1}$ and $R_{2}$ are biased at $-80 \mathrm{~V}$ and $-30 \mathrm{~V}$, respectively. The sweep of the discriminator grid $D$ was set by a computer control and data acquisition program, with a range of typically $\pm 50 \mathrm{~V}$. A battery pack consisting of a series of $9 \mathrm{~V}$ batteries was inserted in the discriminator circuit to offset the range as needed to cover the region around the plasma potential. During the experiments, five batteries provided a sweep range of $V_{B}=[-5-95] \mathrm{V}$. The number of ramp steps for the voltage sweep was set at 300 , and the number of samples per bias step for averaging was set at 200 in order to reduce noise. The collector was biased at $-27 \mathrm{~V}$ with respect to ground. The current signal from the collector was transmitted to an isolated amplifier which amplified it 100 times, then it was digitised and stored to a computer. 
The probe was inserted radially through the $40 \mathrm{CF}$ ConFlat port on the dome at an axial distance of $z=50 \mathrm{~cm}$ from the front of the source chamber. The radial movements of the probe was controlled by an electric step motor, starting at $0 \mathrm{~cm}$ near the inner wall of the dome and moving up to $24 \mathrm{~cm}$ inwards (centre of Njord at $18 \mathrm{~cm}$ ) with step lengths at $1 \mathrm{~cm}$. The position is controlled by the same program as for the discriminator sweep. The RFEA grid configuration and the probe settings were kept at the same values throughout all the experiments, and all experiments involving centre parameter scans were performed with the RFEA placed at $18 \mathrm{~cm}$, equivalent for $r=0$.

In order to estimate the ion density $n_{i}$, the ion velocity distribution function $f_{i}$ must be obtained,

$$
n_{i}=\int f_{i}(v) \mathrm{d} v
$$

The distribution function can be obtained by using the collected current $I_{C}$ in front of the aperture (Stenzel et al. 1982; Chabert \& Braithwaite 2011). $I_{C}$ can be expressed as

$$
I_{C}(v)=A e v n_{i}=A e \int_{v_{M i n}}^{\infty} v f_{i}(v) \mathrm{d} v,
$$

where $e$ is the elemental charge, $v$ the velocity and $A$ is a constant which depends on the front-plate aperture and grid transparency. By considering the direction of the velocity towards the aperture, the minimum velocity $v_{\text {Min }}$ which is required in order to pass $V_{B}$ can be estimated by setting the kinetic energy $E_{K}$ equal to the probe bias potential

$$
\begin{aligned}
E_{K} & =\frac{1}{2} m_{i} v_{M i n}^{2}=e V_{B} \\
& \rightarrow v_{M i n}=\left(\frac{2 e V_{B}}{m_{i}}\right)^{1 / 2} .
\end{aligned}
$$

By substituting $v_{M i n}$ in (3.2) and solving for the potential, one obtains $I_{C}$ as a function of $V_{B}$. Using this, the distribution function for the ions can be obtained from the first derivative of the current as a function of the discriminator potential $I_{C}\left(V_{B}\right)$, given as

$$
f\left(V_{B}\right)=\frac{m_{i}}{A e^{2}} \frac{\mathrm{d} I_{C}\left(V_{B}\right)}{\mathrm{d} V_{B}} .
$$

By using the derivative of $I_{C}$, the distribution function was found in order to extract plasma and ion beam parameters. If no ion beam is formed, or if the RFEA is facing perpendicular to it or is moved radially out of the beam's position, $f\left(V_{B}\right)$ takes the form of a single peak distribution function, where the area underneath the curve represents $n_{i}$ and the peak of $f$ indicates the position of $V_{p}$. This background plasma makes up the tallest peak downstream also when the probe is facing the source, and it is formed mostly by charge-exchange collisions of the source ions with neutrals. In the case when an ion beam is formed and the RFEA is facing directly towards the source, $f\left(V_{B}\right)$ will take the form of a double-peaked distribution function displaying both the background plasma and the ion beam, where the peak on the left side will mark the background plasma and the peak on the right the ion beam. The start of 

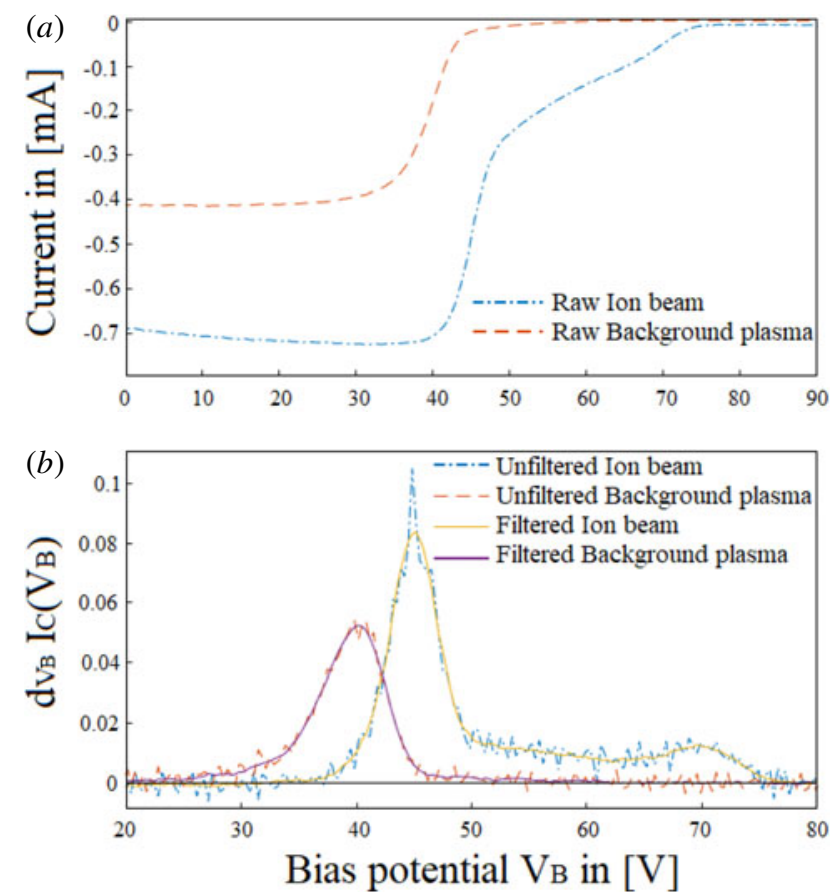

FIguRE 5. (a) Raw data from the collected current $I_{C}$ and (b) raw versus filtered distribution function for an ion beam and background plasma at $400 \mathrm{~W}, P=0.65 \mu \mathrm{Bar}$ and $I_{\text {Coils }}=5$ A, without PMs.

this secondary peak is identified as the point where the derivative of the collector current starts to deviate from the Gaussian slope at $V_{B}>$ peak maximum. This value is chosen because there is not always a distinct minimum between the beam and the background distribution, which one otherwise could have chosen as the starting point. A more precise estimation has been given by Gulbrandsen et al. (2015), where the starting point were chosen as the point where the negative of the double derivative of the collector current was reduced to less than $10 \%$ of its maximum.

In figure 5(a), the raw data obtained from the RFEA show two typical $I_{C}\left(V_{B}\right)$ current profiles, one from a background plasma and the other for an ion beam. The sweep was performed at $400 \mathrm{~W}, P=0.65 \mu$ Bar and $I_{\text {Coils }}=5 \mathrm{~A}$, with the probe faced towards the source. The IV-curve with ion beam was registered at $r=0 \mathrm{~cm}$, and the curve with the background plasma only at $r=18 \mathrm{~cm}$, close to the wall of the plasma chamber. Note that while RFEA current profiles are usually represented as a positive current, the acquisition program in this case displays them as a negative valued current.

Figure $5(b)$ shows the distribution functions obtained by differentiating the $I_{C}\left(V_{B}\right)$ curves in figure $5(a)$ with respect to $V_{B}$. The profile for the background plasma shows a distribution function with a form similar to a Maxwellian distribution function. Often this is not the case for experimental plasmas as they are not always in thermal equilibrium. For the profile with ion beam, the secondary peak on the right-hand side indicates that an ion beam is present. To reduce the noise generated from differentiating, a Savitsky Golay filter with a width of 9 data points (sometimes up to 15) was applied in order to smooth the distribution function. 

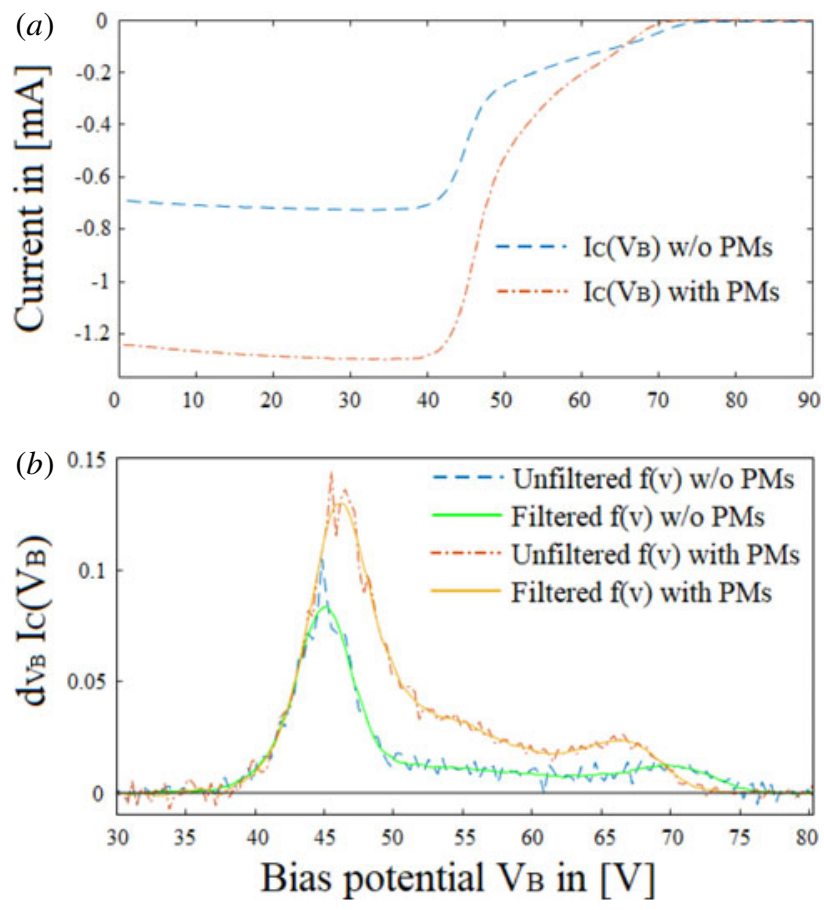

FIGURE 6. (a) Collected current $I_{C}$ and $(b)$ unfiltered and filtered distribution functions for an ion beam at RF-power $400 \mathrm{~W}$, pressure $P=0.65 \mu \mathrm{Bar}$ and coil current $I_{\text {Coils }}=5 \mathrm{~A}$, with and without PMs.

In figure 5(b), the unfiltered and filtered distribution functions are shown. Using the MATLAB function trapz, the area underneath the filtered curve was found and used to obtain the ion density, which was then calibrated against ion densities obtained simultaneously with a Langmuir probe (LP) placed close to the RFEA at $r=0 \mathrm{~cm}$ and $z=50 \mathrm{~cm}$, as shown in figure $1(a)$. To calibrate the density obtained by the RFEA, 30 measurements at various set parameters were obtained simultaneously with the RFEA and a shielded LP.

RFEA probe scans with the PMs on were performed in the same manner, as were the data extractions. Figure $6(a, b)$ shows the raw data and the unfiltered and filtered distribution function with and without PMs respectively, and with the same control parameters as those in figure 5. The comparison of these scans is discussed in further detail in $\S 4$.

Using the characteristics of the distribution function, the plasma potential $V_{p}$, ion density $n_{i}$, beam potential $V_{\text {Beam }}$ and beam energy $E_{\text {Beam }}$ were estimated from the data records. Figure 7(a) illustrates how $n_{i}$ and $V_{p}$ was found from the distribution function for a background plasma only, and figure $7(b)$ illustrates how $V_{p}, V_{\text {Beam }}, E_{\text {Beam }}$ and $n_{\text {Beam }}$ was extracted from the distribution function when an ion beam is present.

\section{Results}

Using the method described in the last section, $V_{p}, V_{\text {Beam }}, n_{i}$ and $E_{\text {Beam }}$ at different RF-powers, pressures and magnetic field strengths were obtained and the data with and without PMs were compared. The results from scans with equal parameter settings 

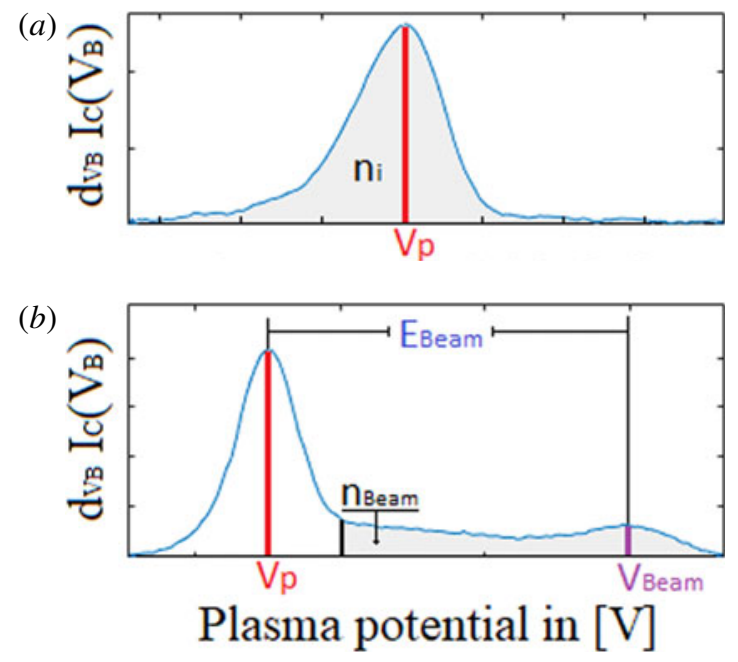

FIGURE 7. (a) Distribution function for a background plasma and (b) with an ion beam.

were compared in order to test the reproducibility of the scans. The parameters differed by as little as $1 \%$ over most of their range, and in some cases up to $\sim 5 \%$.

In the first comparison, two radial profiles of $V_{p}$ and $n_{i}$ at two different RF-powers are given in figures $8(a)$ and $8(b)$ respectively, at a pressure of $0.65 \mu \mathrm{Bar}$ and an axial magnetic field of $\sim[165-170]$ Gauss at a coil current of $I_{\text {Coils }}=5$ A.

Figure $8(a)$ shows that the effect on the plasma potential $V_{p}$ is moderate. At $400 \mathrm{~W}$, $V_{p}$ is slightly higher $(\sim 1 \mathrm{~V})$ in the centre of the column with the cusp field added. At $800 \mathrm{~W}$, it is lower by a similar amount near the edge of the plasma column.

In figure $8(b)$, the radial ion density $n_{i}$ profiles with and without an additional cusp field are shown for RF powers of 400 and $800 \mathrm{~W}$. At $400 \mathrm{~W}$ a moderate increase in density is seen, and a substantial increase can be observed at $800 \mathrm{~W}$, in particular near the centre of the plasma column, where the density increases from approximately $\sim 5.22 \times 10^{16} \mathrm{~m}^{-3}$ to $\sim 1.05 \times 10^{17} \mathrm{~m}^{-3}$.

To better illustrate the effect of the magnetic cusp field on the plasma, the ion density ratio $n_{r}$ is introduced as the ratio between the ion density with the added magnet cusp field $\left(n_{i \mathrm{Mag}}\right)$ and that without it $\left(n_{i}\right)$

$$
n_{r}=\frac{n_{i \mathrm{Mag}}}{n_{i}}
$$

For the remaining data sets, results for the density will be given as $n_{r}$. In figure $8(c)$, $n_{r}$ for the two radial profiles from figure $8(b)$ is shown; $n_{r}$ increases from the outer edge of the plasma column towards the centre until a plateau is reached at approximately $r=8 \mathrm{~cm}$. The same radial behaviour is seen for both low (400 W) and high $(800 \mathrm{~W}) \mathrm{RF}$-powers, with a ratio of about 2 near the centre at $800 \mathrm{~W}$ and approximately 1.8 at $400 \mathrm{~W}$.

In figure $9, n_{r}$ at $r=0 \mathrm{~cm}$ in the RF-power range [100-800] $\mathrm{W}$ is shown. The ratio $n_{r}$ in the centre of the column increases from a moderate factor of 1.2 at low $\mathrm{RF}$-power to a maximum of $\sim 2$ at the RF-power range [600-800] W.

From figure 9 it is clear that, along with an increasing inserted RF-power, the added magnetic cusp field from the PMs causes the plasma density to increase over the 

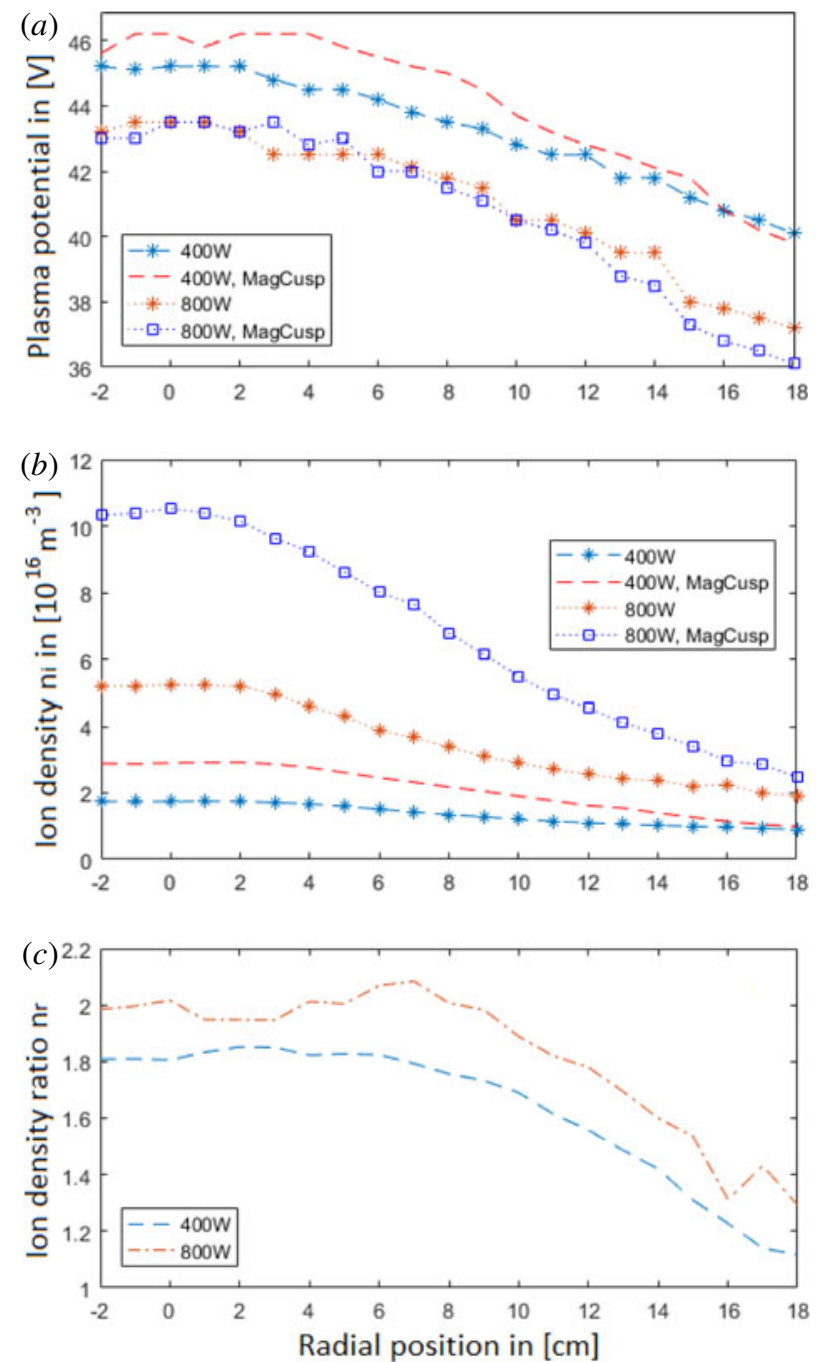

FIGURE 8. Radial profiles of $(a)$ the plasma potential $V_{p},(b)$ the ion density $n_{i}$ and $(c)$ ion density ratio $n_{r}$ versus RF-power at pressure $P=0.65 \mu \mathrm{Bar}$ and magnetic coil current $I_{\text {Coils }}=5 \mathrm{~A}$ at RF-power 400 and $800 \mathrm{~W}$, with and without the magnet band.

entire radial range. The increase in plasma density near the wall is possibly due to the electrons which would have been lost to the wall instead being guided away from it, causing an increase in the plasma density at the edge of the chamber. The largest effect from the PMs is evident in the centre of the chamber where $n_{r}$ at RF powers above $600 \mathrm{~W}$ reached a plateau value of approximately 2 with the added PMs. One possible explanation is that the PMs reflect the plasma charges, consisting of both ions and electrons, radially inwards, causing a build up of the plasma density towards the centre.

How the PMs affect the antenna and the matching network, possibly changing the effective power transfer (EPT), that is, how much power is actually deposited in the plasma, should also be considered. The EPT is not directly obtained with the present experimental set-up. However, a change in EPT should affect the plasma 


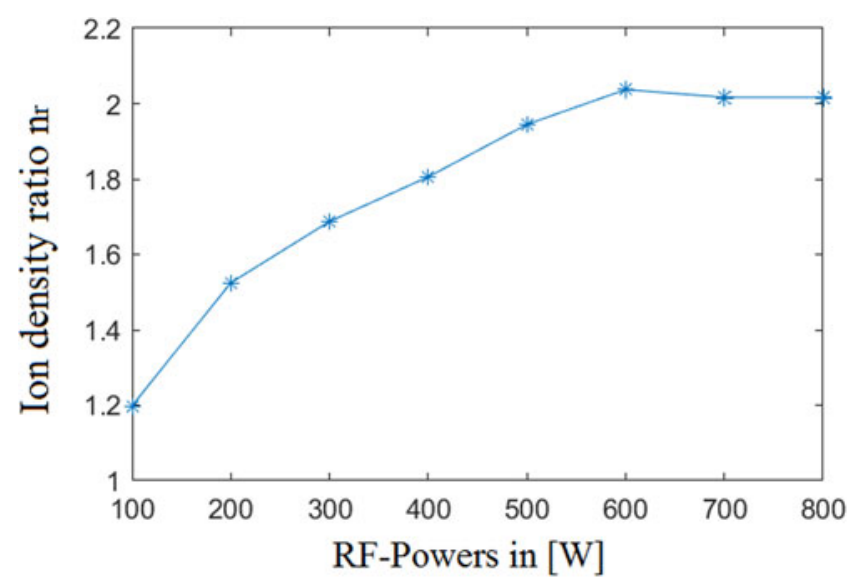

FIgURE 9. Centre density ratio $n_{r}$ versus RF-power at pressure $P=0.65 \mu \mathrm{Bar}$ and magnetic coil current $I_{\text {Coils }}=5 \mathrm{~A}$ at RF-power range [100-800] W.

density in the source. Hence, as a test of this possibility, a Langmuir probe was inserted directly in the source and the ion saturation current was measured at some of the control parameters in order to obtain $n_{i}$ with and without the PMs. The results showed that the difference between the two cases was negligible, i.e. the increase, and sometimes decrease, was less than $4 \%$ of the densities without the PMs. Furthermore, the distance between the placement of the PMs and the antenna is $10 \mathrm{~cm}$ in the axial distance, and the magnetic field from the PMs has a strength of 0.027 Gauss according to the model, a negligible value. The PMs were thus found not to affect the EPT significantly. It was noticed however that reflected power $P_{R e f}$ without retuning would increase by [3-5] W with the PMs attached, showing that the they have a small, but observable, impact on the network. However, during measurements, the antenna was constantly tuned for all parameter changes and prior to each radial sweep in order to keep $P_{R e f}$ as small as possible. On average, the reflected power was of the order of $P_{\text {Ref }}=1 \mathrm{~W}$ and $V S W R=1$, showing that the transmission network was in match. Based on the available data, the reason for the increase in $n_{r}$ towards the centre of the plasma column, and also the increase with increasing RF power towards a plateau above $P_{I n} \sim 600 \mathrm{~W}$, is still elusive. Further work is needed, including also radial measurements at different axial positions, to investigate whether the increase in downstream density is consistent along the $z$-axis as well.

The pressure tests were performed in a pressure range of $P=[0.65-1.05] \mu$ Bar where an ion beam can be detected, at RF-power $400 \mathrm{~W}$, and magnetic coil current $I_{\text {Coils }}=5 \mathrm{~A}$. The radial profiles of $n_{r}$ at three different pressures are shown in figure 10 .

Also in this case, the results show that the cusp field significantly increases the density in the plasma interior. At both $0.85 \mu$ Bar and $1.05 \mu$ Bar the centre density was increased by a factor of approximately 1.9. At the edge of the plasma the density increased from a factor of $\sim 1.1$ at $0.65 \mu$ Bar to a factor slightly over 1.3 at $1.05 \mu$ Bar. While $n_{r}$ in the centre did not increase further from $0.85 \mu$ Bar to $1.05 \mu$ Bar, the ratio at the edge steadily increased at the highest pressures as well. No more data from higher pressures are presented, as the RFEA did not detect an ion beam. 


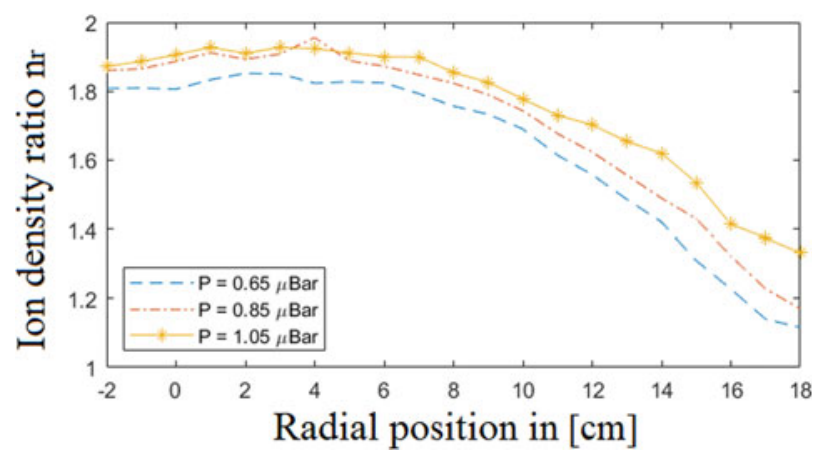

FIGURE 10. Radial ion density ratio versus pressure values $P=0.65 \mu$ Bar at flow $Q=2.0$ SCCM, $P=0.85 \mu$ Bar at flow $Q=3.0 \mathrm{SCCM}$ and $P=1.05 \mu$ Bar at flow $Q=4.0$ SCCM at RF-power $400 \mathrm{~W}$ and magnetic coil current $I_{\text {Coils }}=5 \mathrm{~A}$.

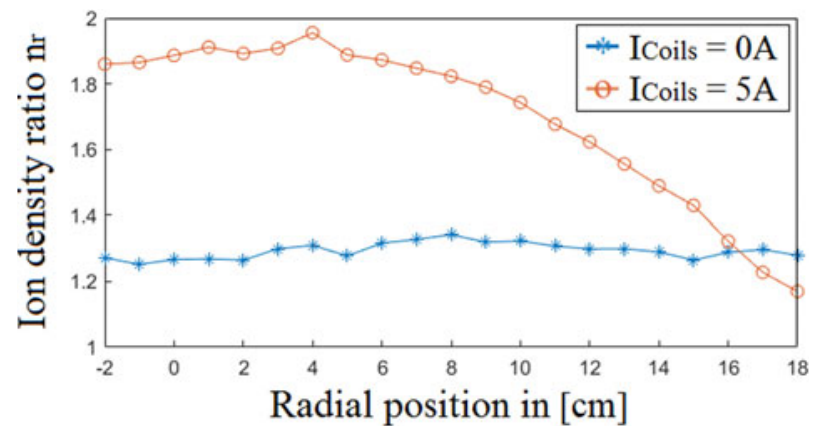

FIgURE 11. Radial ion density ratio $n_{r}$ versus magnetic coil current $I_{\text {Coils }}$ values at 0 and $5 \mathrm{~A}$ at RF-power $=400 \mathrm{~W}$ and pressure $P=0.85 \mu \mathrm{Bar}$ with and without PMs.

The density ratio at different axial magnetic field strengths was obtained at a RF-power of $400 \mathrm{~W}$ and a pressure $P=0.85 \mu$ Bar with a magnetic coil current range $I_{\text {Coils }}=[0-6] \mathrm{A}$ in steps of $1 \mathrm{~A}$, which corresponds to an axial magnetic field strength range of [0-200] Gauss. Two radial profiles at two different axial magnetic field strengths are shown in figure 11.

The scan with no axial magnetic field was conducted in order to study how the cusp field from the PMs alone affects the plasma density, as well as to find a radial profile for $n_{r}$. When no axial magnetic field is present the cusp field increases the overall density by a factor of $\sim 1.3$ over the entire radial range. This suggest that the PMs cause the plasma density to increase nearly evenly over the radial range. In order to see how much the PMs affect the radial plasma density without any axial magnetic field, a new test with more powerful PMs should be conducted. At $I_{\text {Coils }}=5 \mathrm{~A}, n_{r}$ increases to a factor of approximately 1.9 at the centre. At the edge, $n_{r}$ was lower, at $\sim 1.2$, compared to $\sim 1.3$ at $I_{\text {Coils }}=0 \mathrm{~A}$, indicating that the combination of an axial magnetic field and PMs provides a better increase of $n_{r}$.

The ion density in the centre at $r=0 \mathrm{~cm}$ and $z=50 \mathrm{~cm}$ as a function of $I_{\text {Coils }}$ is depicted in figure $12(a)$ and $n_{r}$ is shown in figure $12(b)$.

As depicted, $n_{r}$ reduces to a factor just above 0.8 in the $I_{\text {Coils }}$ range [1-2] A, or [35-70] Gauss, and from figure 12(a), one can see that there is a density peak present 

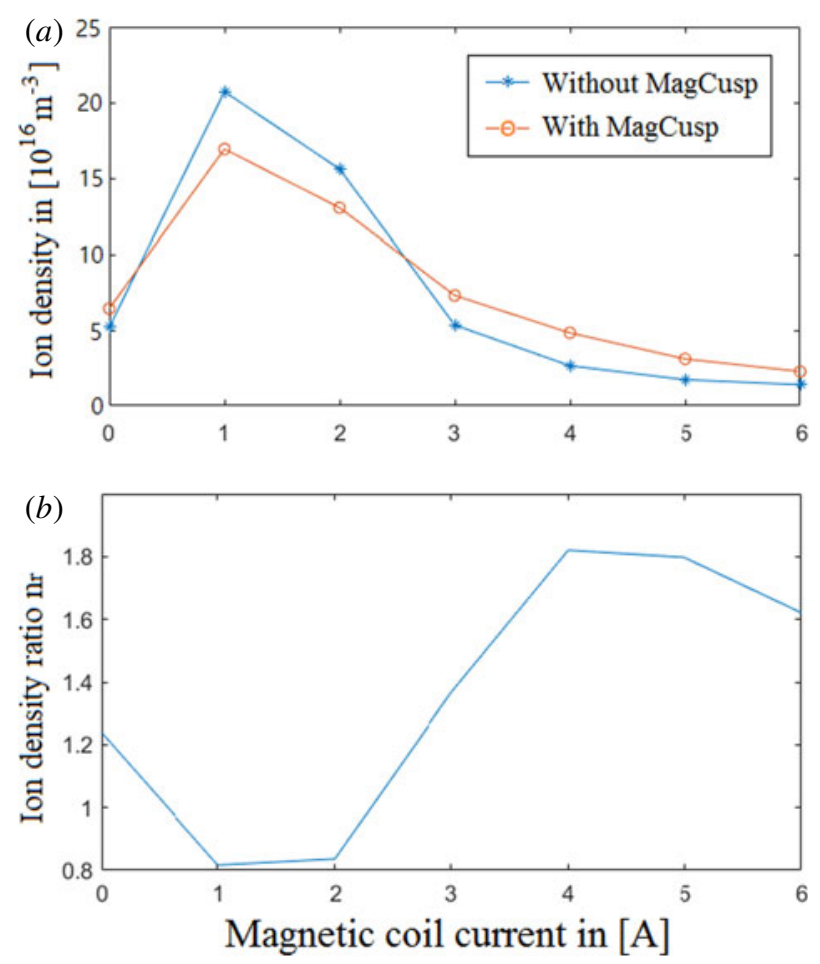

FIGURE 12. Centre ion density $n_{i}$ and ion density ratio $n_{r}$ versus magnetic coil current $I_{\text {Coils }}$ at RF-power $=400 \mathrm{~W}$ and pressure $P=0.85 \mu$ Bar.

at a low axial magnetic field. From the other experiments one would assume that the PMs would improve the magnetic confinement and hence increase the density. However, at a magnetic field strength below $~ 100$ Gauss with the PMs on, $n_{r}$ at this range reduces with the PMs attached. However, no radial probe sweeps were performed at these low $I_{\text {Coils }}$ values in order to obtain radial $n_{r}$ profiles.

Density peaks at low magnetic fields have been noticed earlier in different experiments (Shamrai \& Taranov 1996; Chen 2003; Cho 2006; Lafleur, Charles \& Boswell 2010a,b; Barada et al. 2013; Wang et al. 2015). Studies of this phenomenon have not yet provided an exact physical explanation, but several suggestions point to a helicon mode or a Trivelpiece-Gould (TG) mode which causes the plasma production to increase at a low axial magnetic field. If this is the case, then it appears that the PMs affect this mode in the opposite direction, causing a reduction of the ion density at a low axial magnetic field.

The beam energy $E_{\text {Beam }}$ was found from the difference between the beam potential and the plasma potential as described in figure $7(b)$, and by using energy conservation the beam velocity $v_{\text {Beam }}$ can be estimated,

$$
\left.\begin{array}{c}
E_{\text {Beam }}=e\left(V_{\text {Beam }}-V_{p}\right) \\
E_{k}=\frac{1}{2} m_{i} v_{\text {Beam }}^{2}=e\left(V_{\text {Beam }}-V_{p}\right) \\
v_{\text {Beam }}=\sqrt{\frac{2 e}{m_{i}}\left(V_{\text {Beam }}-V_{p}\right) .}
\end{array}\right\}
$$




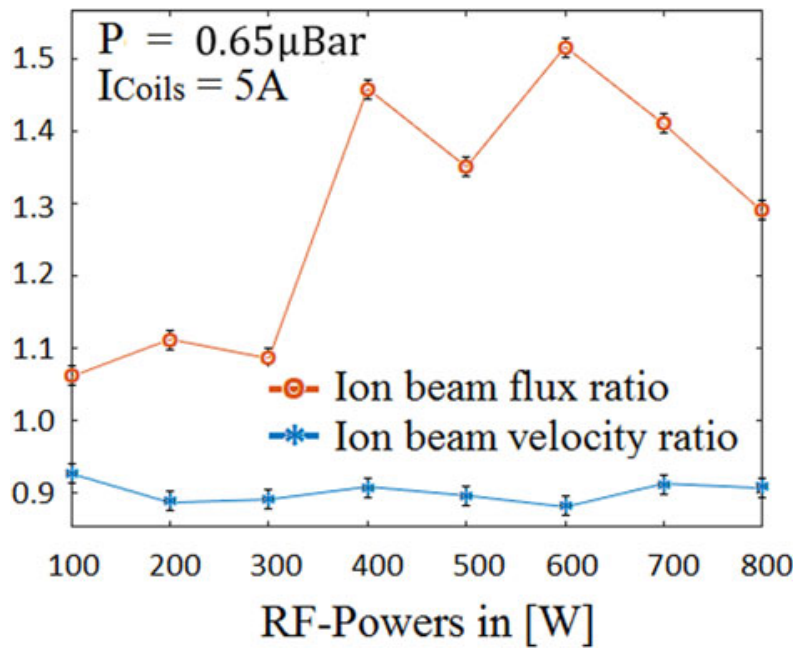

FIGURE 13. Centre ion beam velocity ratio $v_{B r}$ and ion beam flux ratio $\phi_{B r}$ versus RF-power at pressure $P=0.65 \mu$ Bar and magnetic coil current $I_{\text {Coils }}=5 \mathrm{~A}$.

The change in the beam velocity $v_{\text {Beam }}$ was estimated similar to $n_{r}$ by defining the ion beam velocity ratio $v_{B r}$ as the square root of the ratio between the beam energy $E_{\text {BeamMag }}$ with the PMs and the beam energy $E_{\text {Beam }}$ without them

$$
v_{B r}=\sqrt{\frac{E_{\text {BeamMag }}}{E_{\text {Beam }}} .}
$$

Using this ratio, the change in beam velocity was estimated for the different RFpowers, pressures and magnetic field strength values in the cases where an ion beam could be registered. The variations with RF-power are depicted in figure 13, and the ratios as a result of pressure and $I_{\text {Coils }}$-variations are listed in table 1 .

As shown, $v_{B r}$ reduces by a factor of $\sim 0.9$ for all parameter changes, with some small variations. One possible explanation could be that, while we observe that the downstream plasma potential $V_{p}$ increases only slightly, with $\sim 1 \mathrm{~V}$ less, the beam potential $V_{\text {Beam }}$ decreases more significantly (see figure $8 a$ ), a sign that the plasma potential in the source decreases with the PMs attached. The reduced plasma potential can also indicate an increased density (Hopwood et al. 1993). Thus, the beam velocity decreases due to a lower plasma potential in the source. This might be due to a better electron confinement, as the cusp field of the PMs would prevent the electrons from hitting the port wall, which they otherwise do with the purely expanding field (Fredriksen et al. 2010). Upon being reflected from the cusp field, the electrons will move freely upstream back into the source and thus possibly contribute towards a lower plasma potential there.

In order to find how the PMs affects the ion beam density, the ion beam density ratio $n_{B r}$ is introduced as the ratio between the ion beam density with the PMs on, $n_{\text {BeamMag }}$, and without them, $n_{\text {Beam }}$, similar to $n_{r}$ and $v_{B r}$. The beam density was found by measuring the area beneath the secondary peak as showed in figure $7(b)$. By multiplying $n_{B r}$ with $v_{B r}$ from (4.3), the ion beam flux ratio can be defined as

$$
\phi_{B r}=n_{B r} v_{B r}
$$




$\begin{array}{lccc} & v_{B r} & n_{B r} & \phi_{B r} \\ Q(\mathrm{SCCM}) & & & \\ 2 & 0.90 & 1.4598 & 1.313 \\ 3 & 0.89 & 1.6920 & 1.505 \\ 4 & 0.87 & 1.3367 & 1.163 \\ I_{\text {Coils }}(\mathrm{A}) & & & \\ 4 & 0.90 & 1.3515 & 1.216 \\ 5 & 0.89 & 1.6920 & 1.505 \\ 6 & 0.87 & 1.6123 & 1.402\end{array}$

TABLE 1. Estimated values for the ion beam velocity ratio $v_{B r}$, ion beam density ratio $n_{B r}$ and flux ratio $\phi_{r}$ at different flows $Q$ and magnetic coil currents $I_{\text {Coils }}$ at RF-power $400 \mathrm{~W}$, for which a beam is present.

The results of the flux ratio of the beam as a function of RF-power is shown in figure 13, while the results for the pressure and $I_{\text {Coils }}$ variations are listed in table 1 .

As shown, the ion beam flux at $r=0 \mathrm{~cm}$ increased by a factor of 1.5 at each individual parameter setting. Throughout all the estimated $\phi_{r}$ values, it is clear that the flux ratio depends more on the behaviour of the beam density ratio $n_{B r}$, as $v_{B r}$ stays at $\sim 0.9$. The jump in $\phi_{r}$ between 300 and $400 \mathrm{~W}$ can possibly be contributed to a mode change in the plasma when the RF-power increases, causing an increase in the beam density as $n_{r}$ increases.

Given that inductively coupled helicon plasmas have been applied to the development of ion engines, and that particle flux can be used to estimate the thrust (Sutton \& Biblarz 2010), it is possible that permanent magnets, as investigated here, could affect also the thrust of such engines. However, the design of the present experiment is not optimal in order to discuss this possibility further. A more dedicated experiment would be needed in order to decide the possible effects on thrust of RF ion engines.

\section{Conclusions}

The results from the experiments have shown that the application of a magnetic multipole cusp field around the port of a helicon plasma source causes a significant increase in the downstream plasma density. The plasma density increased by a ratio up to 1.8-2 in the centre of the plasma column and up to 1.3 at the plasma edges at different parameter values for the RF-power, pressure and magnetic field strength, separately. Without any axial magnetic field, the applied magnet band provided an increased density just below a factor of 1.3 across the entire radius. A combination of the three variables may increase the density even further, contributing to an increased plasma confinement in the system.

The ion beam velocity was reduced overall by approximately $10 \%$, and due to the increased density, the ion beam flux increased by up to a factor of 1.5 , at each separate parameter change. A combination of these three parameters might increase the flux ratio even further. This effect could possibly be utilised in order to improve the thrust from plasma thrusters.

\section{Acknowledgements}

Author E. Varberg carried out the experiments and analysis, under supervision and assistance by author $\AA$. Fredriksen. The authors want to thank I. Stømmesen and the 
staff at the mechanical workshop for their excellent technical assistance. The authors wish to thank the referees who kindly reviewed the article and gave insightful and constructive comments and questions during the publication process. This research was supported and entirely funded by the department of Physics and Technology at UiT The Arcitic Universy of Norway.

\section{REFERENCES}

Barada, K., Chattopadhyay, P., Ghosh, J., Kumar, S. \& Saxena, Y. 2013 Observation of low magnetic field density peaks in helicon plasma. Phys. Plasmas 20 (4), 042119.

Byhring, H., Charles, C., Fredriksen, Å.\& Boswell, R. 2008 Double layer in an expanding plasma: simultaneous upstream and downstream measurements. Phys. Plasmas 15 (10), 102113.

Chabert, P. \& Braithwaite, N. 2011 Physics of Radio-Frequency Plasmas. Cambridge University Press.

ChARLes, C. 2004 Hydrogen ion beam generated by a current-free double layer in a helicon plasma. Appl. Phys. Lett. 84 (3), 332-334.

Charles, C. 2007 A review of recent laboratory double layer experiments. Plasma Sources Sci. Technol. 16 (4), R1.

Charles, C. 2009 Plasmas for spacecraft propulsion. J. Phys. D: Appl. Phys. 42 (16), 163001.

Charles, C. \& Boswell, R. W. 2004 Laboratory evidence of a supersonic ion beam generated by a current-free 'helicon' double-layer. Phys. Plasmas 11 (4), 1706-1714.

CHEN, F. F. 2008 Permanent magnet helicon source for ion propulsion. IEEE Trans. Plasma Sci. 36 (5), 2095-2110.

Chen, F. F. 2003 The low-field density peak in helicon discharges. Phys. Plasmas 10 (6), 2586-2592.

Chen, F. F. 2012 Performance of a permanent-magnet helicon source at 27 and $13 \mathrm{mHz}$. Phys. Plasmas 19 (9), 093509.

Chen, F. F. 2014 Helicon discharges and sources: a review. Plasma Sources Sci. Technol. 24 (1), 014001.

Chen, F. F. \& Torreblanca, H. 2009 Permanent-magnet helicon sources and arrays: a new type of rf plasma. Phys. Plasmas 16 (5), 057102.

Сно, S. 2006 The resistance peak of helicon plasmas at low magnetic fields. Phys. Plasmas 13 (3), 03350 .

Cohen, S. A., Sun, X., Ferraro, N. M., Scime, E. E., Miah, M., Stange, S., Siefert, N. S. \& BoIvin, R. F. 2006 On collisionless ion and electron populations in the magnetic nozzle experiment (mnx). IEEE Trans. Plasma Sci. 34 (3), 792-803.

Corr, C., Zanger, J., Boswell, R. \& Charles, C. 2007 Ion beam formation in a low-pressure geometrically expanding argon plasma. Appl. Phys. Lett. 91 (24), 241501.

Ebersohn, F. H., Sheehan, J., Longmier, B. W. \& Shebalin, J. V. 2014 Quasi-one-dimensional code for particle-in-cell simulation of magnetic nozzle expansion. In PROC 50th AIAA/ASME/SAE/ASEE Joint Propultion Conference July 28-30, 2 2014, Cleveland, OH.

Fredriksen, Å., MishrA, L. \& B YHring, H. 2010 The effects of downstream magnetic field on current-free double layers and beam formation in the njord helicon plasma device. Plasma Sources Sci. Technol. 19 (3), 034009.

GulbrandSEN, N. \& Fredriksen, $\AA 2017$ RFEA measurements of high-energy electrons in a helicon plasma device with expanding magnetic field. Front. Phys. 5, 2.

Gulbrandsen, N., Fredriksen, A., CARR, J. JR. \& Scime, E. 2015 A comparison of ion beam measurements by retarding field energy analyzer and laser induced fluorescence in helicon plasma devices. Phys. Plasmas 22 (3), 033505.

Hopwood, J., Guarnieri, C., Whitehair, S. \& CuOMo, J. 1993 Electromagnetic fields in a radio frequency induction plasma. J. Vac. Sci. Technol. A11, 147.

Hutchinson, I. 1987 Principles of Plasma Diagnostics. Cambridge University Press.

Lafleur, T., Charles, C. \& Boswell, R. W. 2010a Plasma control by modification of helicon wave propagation in low magnetic fields. Phys. Plasmas 17 (7), 073508. 
Lafleur, T., Charles, C. \& Boswell, R. W. $2010 \mathrm{~b}$ Ion beam formation in a very low magnetic field expanding helicon discharge. Phys. Plasmas 17 (4), 043505.

Leung, K., Collier, R., Marshall, L., Gallaher, T., Ingham, W., Kribel, R. \& Taylor, G. 1978 Characteristics of a multidipole ion source. Rev. Sci. Instrum. 49 (3), 321-325.

Lieberman, M. \& Lichtenberg, A. 2005 Principles of Plasma Discharges and Materials Processing, 2nd edn. Wiley-Interscience.

OHKAWA, T. \& Kerst, W. 1961 Multipole magnetic field configurations for stable plasma confinement. Il Nuovo Cimento 22 (4), 784-799.

Olsen, C. S., Ballenger, M. G., Carter, M. D., Diaz, F. R. C., Giambusso, M., Glover, T. W., Ilin, A. V., SQuire, J. P., Longmier, B. W., Bering, E. A. et al. 2015 Investigation of plasma detachment from a magnetic nozzle in the plume of the vx-200 magnetoplasma thruster. IEEE Trans. Plasma Sci. 43 (1), 252-268.

Schoenberg, K. F., Gerwin, R. A., Henins, I., Mayo, R. M., Scheuer, J. T. \& Wurden, G. A. 1993 Preliminary investigation of power flow and performance phenomena in a multimegawatt coaxial plasma thruster. IEEE Trans. Plasma Sci. 21 (6), 625-644.

Shamrai, K. P. \& TARAnov, V. B. 1996 Volume and surface RF power absorption in a helicon plasma source. Plasma Sources Sci. Technol. 5 (3), 474-491.

Stenzel, R., Williams, R., Agüero, R., Kitazaki, K., Ling, A., McDonald, T. \& Spitzer, J. 1982 Novel directional ion energy analyzer. Rev. Sci. Instrum. 53 (7), 1027.

Sutton, G. \& Biblarz, O. 2010 Rocket Propulsion Elements, 8th edn. John Wiley \& Sons, Inc.

TAKAHASHI, K. \& ANDO, A. 2017 Laboratory observation of a plasma-flow-state transition from diverging to stretching a magnetic nozzle. Phys. Rev. Lett. 118 (22), 225002.

TAKAHASHI, K., ITOH, Y. \& FuJIWARA, T. 2011 Operation of a permanent-magnets-expanding plasma source connected to a large-volume diffusion chamber. J. Phys. D: Appl. Phys. 44 (1), 015204.

Takahashi, K., Oguni, K., Yamada, H. \& Fujiwara, T. 2008 Ion acceleration in a solenoid-free plasma expanded by permanent magnets. Phys. Plasmas 15 (8), 084501.

TAKahashi, K., Shida, Y. \& Fujiwara, T. 2010 Magnetic-field-induced enhancement of ion beam energy in a magnetically expanding plasma using permanent magnets. Plasma Sources Sci. Technol. 19 (2), 025004.

Takahashi, K., Shida, Y., Fujinara, T. \& Oguni, K. 2009 Supersonic ion beam driven by permanent-magnets-induced double layer in an expanding plasma. IEEE Trans. Plasma Sci. 37 (8), 1532-1536.

Virko, Y., Virko, V., Shamrai, K. \& Yakimenko, A. 2007 Characterization of plasma and emergent ion beam in a compact helicon source with permanent magnets. Prblms. Atomic Sci. Technol. 38 (41), 136-138.

Wang, Y., Zhao, G., LiU, Z. W., Ouyang, J. T. \& Chen, Q. 2015 Two density peaks in low magnetic field helicon plasma. Phys. Plasmas 22 (9), 093507.

YUN, S., KIM, J. \& CHANG, H. 1998 Electrical characteristics of helicon wave plasmas. IEEE Trans. Plasma Sci. 26 (2), 159-166. 